Djairo G. de Figueiredo · Jean-Pierre Gossez · Pedro Ubilla

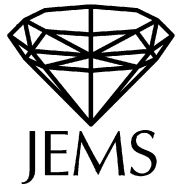

\title{
Multiplicity results for a family of semilinear elliptic problems under local superlinearity and sublinearity
}

Received June 13, 2005 and in revised form November 7, 2005

\begin{abstract}
We study the existence, nonexistence and multiplicity of positive solutions for the family of problems $-\Delta u=f_{\lambda}(x, u), u \in H_{0}^{1}(\Omega)$, where $\Omega$ is a bounded domain in $\mathbb{R}^{N}, N \geq 3$ and $\lambda>0$ is a parameter. The results include the well-known nonlinearities of the Ambrosetti-Brezis-Cerami type in a more general form, namely $\lambda a(x) u^{q}+b(x) u^{p}$, where $0 \leq q<1<p \leq 2^{*}-1$. The coefficient $a(x)$ is assumed to be nonnegative but $b(x)$ is allowed to change sign, even in the critical case. The notions of local superlinearity and local sublinearity introduced in [9] are essential in this more general framework. The techniques used in the proofs are lower and upper solutions and variational methods.
\end{abstract}

Keywords. Multiplicity, semilinear elliptic problem, local sub- and superlinear nonlinearities, concave-convex nonlinearities, critical exponent, upper and lower solutions, variational method

\section{Introduction}

This paper is concerned with the existence, nonexistence and multiplicity of solutions for the family of problems

$$
\begin{cases}-\Delta u=f_{\lambda}(x, u) & \text { in } \Omega, \\ u>0 & \text { in } \Omega, \\ u=0 & \text { on } \partial \Omega,\end{cases}
$$

where $\Omega$ is a bounded domain in $\mathbb{R}^{N}, N \geq 3$, and $\lambda>0$ is a parameter. An important feature of this family is its monotone dependence on $\lambda$, i.e. $f_{\lambda}(x, s) \leq f_{\lambda^{\prime}}(x, s)$ if $\lambda<\lambda^{\prime}$.

Research of D. G. de Figueiredo supported by CNPq-FNRS, PRONEX, FAPESP; of J.-P. Gossez by CNPq-FNRS; and of P. Ubilla by FONDECYT 1040990.

D. G. de Figueiredo: IMECC-UNICAMP. Caixa Postal 6065, 13081-970 Campinas, SP, Brasil; e-mail: djairo@ime.unicamp.br

J.-P. Gossez: Département de Mathématique, C.P. 214, Université Libre de Bruxelles, 1050 Bruxelles, Belgique; e-mail: gossez@ulb.ac.be

P. Ubilla: Universidad de Santiago de Chile, Casilla 307, Correo 2, Santiago, Chile; e-mail:pubilla@usach.cl

Mathematics Subject Classification (2000): 35J20, 35 J60 
There are several motivations to our study. One of them comes from the following example:

$$
\begin{cases}-\Delta u=\lambda a(x) u^{q}+b(x) u^{p} & \text { in } \Omega, \\ u>0 & \text { in } \Omega, \\ u=0 & \text { on } \partial \Omega,\end{cases}
$$

where $0 \leq q<1<p$. This example was extensively studied in [1] when $a(x) \equiv 1$, $b(x) \equiv 1$; it was in particular shown there that if $p \leq 2^{*}-1$ where $2^{*}=2 N /(N-2)$, then there exists $0<\Lambda<\infty$ such that (1.2) has at least two solutions for $\lambda<\Lambda$, at least one solution for $\lambda=\Lambda$, and no solution for $\lambda>\Lambda$. In this paper we extend this result of [1] to the case of variable coefficients $a(x)$ and $b(x)$, with $a(x) \geq 0$ but $b(x)$ possibly indefinite. This is partly carried out along the lines of our previous work [9] where the notions of local superlinearity and local sublinearity were introduced. The main difference here with respect to [9], as far as example (1.2) is concerned, is the assumption $a(x) \geq 0$ in $\Omega$. This allows in particular the use of the strong maximum principle. We emphasize that $b(x)$ in $(1.2)$ is allowed to change sign even in the critical case where $p=2^{*}-1$. As observed in [5, p. 454], critical problems become more delicate in the presence of variable coefficients. In this respect, our basic assumption on $b(x)$ above in the critical case requires that $b(x)$ remains equal or sufficiently close to $\|b\|_{\infty}$ on a small ball (cf. condition $(b)$ in Theorem 4.2).

Our results relative to (1.1) apply as well to several situations rather different from example (1.2). We can handle for instance a problem like

$$
\begin{cases}-\Delta u=\lambda c(x)(u+1)^{p} & \text { in } \Omega, \\ u>0 & \text { in } \Omega, \\ u=0 & \text { on } \partial \Omega,\end{cases}
$$

where $1<p \leq 2^{*}-1$ and $c(x) \geq 0$. This problem was studied in [5] and [11] when $c(x) \equiv 1$.

Our present approach to obtain multiple solutions to (1.1) is different from that in [9]. We follow here the classical method of obtaining a first solution via upper-lower solutions and a second one via the mountain pass theorem. The $H^{1}$ versus $C^{1}$ minimization result of [6] plays an important role in this approach. In the critical case we use some of the techniques developed in [5] and [1] to handle the (PS) condition.

Our results relative to 1.1 are stated in detail in Section 2 and their proofs given in Section 3. Their application to problems (1.2) and (1.3) is dealt with in Section 4.

\section{Statement of results}

In this section we state our results relative to (1.1), first for a nonlinearity of arbitrary growth, then in the subcritical case, and finally in the critical case.

Let $\Omega$ be a smooth bounded domain in $\mathbb{R}^{N}, N \geq 3$. Our general assumption on the family $f_{\lambda}(x, s)$ is: 
(H) For each $\lambda>0, f_{\lambda}: \Omega \times[0, \infty) \rightarrow \mathbb{R}$ is a Carathéodory function with the property that for any $s_{0}>0$, there exists a constant $A$ such that

$$
\left|f_{\lambda}(x, s)\right| \leq A
$$

for a.e. $x \in \Omega$ and all $s \in\left[0, s_{0}\right]$. Moreover if $\lambda<\lambda^{\prime}$, then $f_{\lambda}(x, s) \leq f_{\lambda^{\prime}}(x, s)$ for a.e. $x \in \Omega$ and all $s \geq 0$.

The following assumption concerns the behavior of $f_{\lambda}(x, s)$ near $s=0$; it implies $f_{\lambda}(x, 0) \geq 0$ and, as assumption $(H)$, will be assumed throughout the paper:

$\left(H_{0}\right)$ For each $\lambda>0$ and each $s_{0}>0$, there exists $B>0$ such that

$$
f_{\lambda}(x, s) \geq-B s
$$

for a.e. $x \in \Omega$ and all $s \in\left[0, s_{0}\right]$.

We will always understand that $f_{\lambda}(x, s)$ has been extended for $s<0$ by putting $f_{\lambda}(x, s)=f_{\lambda}(x, 0)$ for $\lambda>0$, a.e. $x \in \Omega$ and $s<0$.

Observe that, at this stage, if $u \in H_{0}^{1}(\Omega) \cap L^{\infty}(\Omega)$ satisfies the equation $-\Delta u=$ $f_{\lambda}(x, u)$ in the $H_{0}^{1}(\Omega)$ sense, then $(H)$ and the standard regularity theory imply $u \in$ $W^{2, r}(\Omega)$ for any $r<\infty$ and so $u \in C^{1}(\bar{\Omega})$. Moreover $u \geq 0$ (in fact, take $-u^{-}$as a test function in the equation and use $\left.f_{\lambda}(x, 0) \geq 0\right)$; in addition, we have $u>0$ in $\Omega$ and $\partial u / \partial v<0$ on $\partial \Omega$ if $u \not \equiv 0$ (this follows from $\left(H_{0}\right)$ and the strong maximum principle). Here $v$ denotes the exterior normal. Observe also that the associated functional

$$
I_{\lambda}(u):=\frac{1}{2} \int_{\Omega}|\nabla u|^{2}-\int_{\Omega} F_{\lambda}(x, u),
$$

where $F_{\lambda}(x, s):=\int_{0}^{s} f_{\lambda}(x, t) d t$, is well defined for $u \in H_{0}^{1}(\Omega) \cap L^{\infty}(\Omega)$.

The following two assumptions will be used in our first result:

$\left(H_{e}\right)$ There exist $\lambda>0$ and a nondecreasing function $g$ with $\inf \{g(s) / s: s>0\}<$ $1 /\|e\|_{\infty}$ such that

$$
f_{\lambda}(x, s) \leq g(s)
$$

for a.e. $x \in \Omega$ and all $s \geq 0$; here $e$ is the solution of $-\Delta e=1$ in $\Omega, e=0$ on $\partial \Omega$, and \|\|$_{\infty}$ denotes the $L^{\infty}(\Omega)$ norm.

$\left(H_{\Omega_{1}}\right)$ For any $\lambda>0$ there exists a smooth subdomain $\Omega_{1}, s_{1}>0$ and $\theta_{1}>\lambda_{1}\left(\Omega_{1}\right)$ such that

$$
f_{\lambda}(x, s) \geq \theta_{1} s
$$

for a.e. $x \in \Omega_{1}$ and all $s \in\left[0, s_{1}\right]$; here $\lambda_{1}\left(\Omega_{1}\right)$ denotes the principal eigenvalue of $-\Delta$ on $H_{0}^{1}\left(\Omega_{1}\right)$.

Here are some comments on the above two assumptions. Assumption $\left(H_{e}\right)$ is a rather standard condition to guarantee the existence of an upper solution (cf. e.g. [10]). This condition is motivated by the fact that an upper solution for an equation of the type $-\Delta u=f(u)$ can be obtained if one has an upper solution for another equation of the 
form $-\Delta u=g(u)$ with $f(s) \leq g(s)$ for all $s$. Assumption $\left(H_{\Omega_{1}}\right)$ is a local sublinearity condition at 0 , which is satisfied for instance if the following stronger condition holds:

$$
\lim _{\substack{s \rightarrow 0 \\ s>0}} \frac{f_{\lambda}(x, s)}{s}=\infty,
$$

uniformly for $x \in \Omega_{1}$. Assumption $\left(H_{\Omega_{1}}\right)$ is used to construct a lower solution.

Theorem 2.1 (Existence of one solution without growth condition). Under the assumptions $(H),\left(H_{0}\right),\left(H_{e}\right)$ and $\left(H_{\Omega_{1}}\right)$, there exists $0<\Lambda \leq \infty$ such that problem (1.1) has at least one solution $u$ (with $I_{\lambda}(u)<0$ ) for $0<\lambda<\Lambda$ and no solution for $\lambda>\Lambda$.

We remark that in the present generality, $\Lambda$ can be $\infty$. One trivial example is provided by a family as above such that, for each $\lambda>0$, there exists $M_{\lambda}>0$ with $f_{\lambda}\left(x, M_{\lambda}\right)<0$ for a.e. $x$. In this case the constant $M_{\lambda}$ is an upper solution.

Theorem 2.2 (Nonexistence for $\lambda$ large). In addition to the hypotheses of Theorem 2.1. assume:

$\left(H_{\tilde{\Omega}}\right)$ There exist a function $h$ with $h(\lambda) \rightarrow \infty$ as $\lambda \rightarrow \infty$, a smooth subdomain $\tilde{\Omega}$ and $\tilde{m} \in L^{\infty}(\tilde{\Omega})$ with $\tilde{m} \geq 0, \tilde{m} \not \equiv 0$, such that

$$
f_{\lambda}(x, s) \geq h(\lambda) \tilde{m}(x) s
$$

for all $\lambda>0$, a.e. $x \in \tilde{\Omega}$ and all $s \geq 0$.

Then $\Lambda<\infty$.

Assumption $\left(H_{\tilde{\Omega}}\right)$ can be looked at as a localized version of the trivial sufficient condition of nonexistence for $-\Delta u=l(u)$ in $\Omega, u>0$ in $\Omega, u=0$ on $\partial \Omega$, namely that $\inf \{l(s) / s$ : $s>0\}>\lambda_{1}(\Omega)$.

Due to the absence of growth condition, we have up to now defined a solution as a function in $H_{0}^{1}(\Omega) \cap L^{\infty}(\Omega)$. However, if the following growth condition with respect to $s$ in the nonlinearity $f_{\lambda}(x, s)$ is assumed, then one can speak of an $H_{0}^{1}(\Omega)$ solution in the usual sense:

(G) For any $[r, R] \subset\{\lambda>0\}$, there exist $d_{1}, d_{2}$ and $\sigma \leq 2^{*}-1$ such that

$$
\left|f_{\lambda}(x, s)\right| \leq d_{1}+d_{2} s^{\sigma}
$$

for all $\lambda \in[r, R]$, a.e. $x \in \Omega$ and all $s \geq 0$.

If $\sigma<2^{*}-1$ in $(G)$, then a standard bootstrap argument gives that any $u \in H_{0}^{1}(\Omega)$ which solves $-\Delta u=f_{\lambda}(x, u)$ belongs to $W^{2, r}(\Omega)$ for any $r<\infty$ and consequently to $C^{1}(\bar{\Omega})$. This conclusion also holds if $\sigma$ in $(G)$ is equal to $2^{*}-1$, by using a result of [4]. Condition $(G)$ (with $\sigma \leq 2^{*}-1$ ) also implies that the functional $I_{\lambda}(u)$ is well defined for $u \in H_{0}^{1}(\Omega)$.

Aiming now to prove the existence of a solution for $\lambda=\Lambda$, we will assume the following condition: 
$(A R)_{d}$ For any $[r, R] \subset\{\lambda>0\}$, there exist $\theta>2, \rho<2, d \geq 0$ and $s_{0} \geq 0$ such that

$$
\theta F_{\lambda}(x, s) \leq s f_{\lambda}(x, s)+d s^{\rho}
$$

for all $\lambda \in[r, R]$, a.e. $x \in \Omega$ and all $s \geq s_{0}$.

This condition $(A R)_{d}$ is a weakening of the classical superquadraticity condition of Ambrosetti-Rabinowitz [2]. It was introduced in [9] in order to handle indefinite nonlinearities.

Theorem 2.3 (Existence of one solution for $\lambda=\Lambda$ ). In addition to the hypotheses of Theorem 2.2. assume $(G),(A R)_{d}$ and the continuity of $f_{\lambda}(x, s)$ with respect to $\lambda$ (for a.e. $x$ and uniformly for $s$ bounded). Then problem (1.1) has at least one solution $u$ (with $\left.I_{\lambda}(u) \leq 0\right)$ for $\lambda=\Lambda$.

Remark. The uniformity with respect to $\lambda \in[r, R]$ in $(G)$ and $(A R)_{d}$ is used only in Theorem 2.3 to deal with the limiting case $\lambda=\Lambda$. It is not needed in the following Theorems 2.4 2.6, where $\lambda<\Lambda$ will be fixed.

Now we discuss multiplicity for subcritical families, namely the ones satisfying $(G)$ with $\sigma<2^{*}-1$. Our purpose is to prove the existence of at least two solutions when $\lambda<\Lambda$. For that matter we have to strengthen a little bit some of the hypotheses of Theorem 2.1. Condition $\left(H_{0}\right)$ is replaced by

$\left(H_{0}\right)^{\prime}$ For any $\lambda>0$ and any $s_{0}>0$, there exists $B \geq 0$ such that for a.e. $x \in \Omega$,

$$
s \mapsto f_{\lambda}(x, s)+B s
$$

is nondecreasing on $\left[0, s_{0}\right]$; moreover $f_{\lambda}(x, 0) \geq 0$ for all $\lambda>0$ and a.e. $x \in \Omega$.

Condition $\left(H_{0}\right)^{\prime}$ is a classical requirement when dealing with upper-lower solutions. The monotonicity of the family $f_{\lambda}$ is also assumed to be strict in the following sense:

(M) For any $\lambda<\lambda^{\prime}$ and any $u \in C_{0}^{1}(\bar{\Omega})$ with $u>0$ in $\Omega$,

$$
f_{\lambda}(x, u(x)) \leq \not \equiv f_{\lambda^{\prime}}(x, u(x))
$$

We will also assume:

$\left(H_{\Omega_{2}}\right)$ For any $\lambda>0$, there exist a subdomain $\Omega_{2}, s_{2}$ and $\theta_{2}>0$ such that

$$
F_{\lambda}(x, s) \geq \theta_{2} s^{2}
$$

for a.e. $x \in \Omega_{2}$ and all $s \geq s_{2}$.

Condition $\left(H_{\Omega_{2}}\right)$ is implied by a local superlinearity condition at $\infty$ of the form

$$
\lim _{s \rightarrow \infty} \frac{f_{\lambda}(x, s)}{s}=\infty
$$

uniformly for $x \in \Omega_{2}$. It is used in conjunction with $(A R)_{d}$ to derive the geometry of the mountain pass. 
Theorem 2.4 (Existence of a second solution in the subcritical case). In addition to the hypotheses of Theorem 2.1, assume $(G)$ with $\sigma<2^{*}-1$ as well as $(A R)_{d},\left(H_{0}\right)^{\prime},(M)$ and $\left(H_{\Omega_{2}}\right)$. Then problem (1.1) has at least two solutions $u$, $v$ for $0<\lambda<\Lambda$, with $u<v$ in $\Omega$, $\partial u / \partial v>\partial v / \partial v$ on $\partial \Omega$ and $I_{\lambda}(u)<0$.

Finally, we consider multiplicity for critical families. This means that $f_{\lambda}(x, s)$ behaves at $\infty$ like $b(x) s^{p}$ with $p=2^{*}-1$. We thus write the function $f_{\lambda}$ as

$$
f_{\lambda}(x, s)=h_{\lambda}(x, s)+b(x) s^{p}
$$

and we distinguish two cases: (i) $h_{\lambda}$ satisfies $(G)$ with $\sigma<1, b(x)$ may change sign, (ii) $h_{\lambda}$ satisfies $(G)$ with $\sigma<2^{*}-1, b(x) \geq 0$ in $\Omega$.

We first deal with case (i).

Theorem 2.5 (Existence of a second solution in the critical case with $\sigma<1$ ). In addition to the hypotheses of Theorem 2.1. assume that $f_{\lambda}(x, s)$ satisfies $\left(H_{0}\right)^{\prime}$ and $(M)$. Suppose also that $f_{\lambda}(x, s)$ can be written as in 2.1) with $p=2^{*}-1, h_{\lambda}(x, s)$ satisfying $(G)$ with $\sigma<1$, and $h_{\lambda}(x, s)$ nondecreasing with respect to $s$ for any $\lambda>0$ and a.e. $x$. Suppose also that $b(x)$ in $(2.1)$ is $\not \equiv 0$, belongs to $L^{\infty}(\Omega)$ and satisfies

(b) for some $x_{0} \in \Omega$, some ball $B_{1} \subset \Omega$ around $x_{0}$, some constant $M$ and some $\gamma$ with $\gamma>2^{*}$ when $N \geq 5, \gamma \geq 2^{*}$ when $N=4, \gamma>3 / 5$ when $N=3$, one has

$$
0 \leq\|b\|_{\infty}-b(x) \leq M\left|x-x_{0}\right|^{\gamma}
$$

for a.e. $x \in B_{1}$. (Recall that \|\|$_{\infty}$ denotes the $L^{\infty}(\Omega)$ norm.)

Then the conclusion of Theorem 2.4 holds.

Assumption $(b)$ implies $\left\|b^{-}\right\|_{\infty} \leq\left\|b^{+}\right\|_{\infty}$, with in addition some limitation on the way $b(x)$ approaches $\|b\|_{\infty}$. It trivially holds if $b(x)=\|b\|_{\infty}$ a.e. on a small ball.

We now deal with the critical case (ii).

Theorem 2.6 (Existence of a second solution in the critical case with $\sigma<2^{*}-1$ ). In addition to the hypotheses of Theorem 2.1. assume that $f_{\lambda}(x, s)$ satisfies $\left(H_{0}\right)^{\prime}$ and $(M)$. Suppose also that $f_{\lambda}(x, s)$ can be written as in (2.1) with $p=2^{*}-1, h_{\lambda}(x, s)$ satisfying $(G)$ with $\sigma<2^{*}-1, h_{\lambda}(x, s)$ nondecreasing with respect to $s$ for any $\lambda>0$ and a.e. $x$, and $h_{\lambda}(x, s)$ satisfying $(A R)_{d}$. Suppose that $b$ in $(2.1)$ is $\not \equiv 0, \geq 0$ in $\Omega$, belongs to $L^{\infty}(\Omega)$ and satisfies condition $(b)$ above. Then the conclusion of Theorem 2.4 holds.

In Theorem 2.6 $h_{\lambda}(x, s)$ is allowed any subcritical growth, at the expense of assuming $(A R)_{d}$ for $h_{\lambda}(x, s)$ and $b(x) \geq 0$. 


\section{Proofs}

This section is devoted to the proofs of all theorems stated above. It will be convenient from now on to denote (1.1) as $(1.1)_{\lambda}$.

Proof of Theorem 2.1. We start by proving the existence of an upper solution of (1.1) $\lambda$ for the value of $\lambda$ provided by $\left(H_{e}\right)$. The construction is inspired from [3] (see also [1], [10]). One takes the solution $e$ of $-\Delta e=1$ in $\Omega, e=0$ on $\partial \Omega$. With $\lambda$ and $g$ given by $\left(H_{e}\right)$, there exists $M>0$ such that

$$
1 /\|e\|_{\infty} \geq g\left(M\|e\|_{\infty}\right) /\left(M\|e\|_{\infty}\right)
$$

and so one has

$$
-\Delta(M e)=M \geq g\left(M\|e\|_{\infty}\right) \geq g(M e) \geq f_{\lambda}(x, M e) .
$$

This shows that $M e$ is a classical upper solution of $(1.1)_{\lambda}$.

We now construct a lower solution for $(1.1)_{\lambda}$ by using the subdomain $\Omega_{1}$ provided by $\left(H_{\Omega_{1}}\right)$. Denote by $\varphi_{1}$ the positive principal eigenfunction of $-\Delta$ on $H_{0}^{1}\left(\Omega_{1}\right)$. Extend $\varphi_{1}$ by 0 on $\Omega \backslash \Omega_{1}$; the extended function, still denoted by $\varphi_{1}$, belongs to $H_{0}^{1}(\Omega) \cap L^{\infty}(\Omega)$. One then argues as in [9, pp. 464-465] to show that for $\varepsilon>0$ sufficiently small, $\varepsilon \varphi_{1}$ is a weak lower solution of $(1.1)_{\lambda}$ which satisfies $\varepsilon \varphi_{1} \leq M e$ in $\Omega$.

It follows that Theorem 2.4 of [13] can be applied; it yields the existence of a solution $u \in H_{0}^{1}(\Omega) \cap L^{\infty}(\Omega)$ of $(1.1)_{\lambda}$ for the value of $\lambda$ provided by $\left(H_{e}\right)$. So at this stage we have proved that

$$
\Lambda:=\sup \left\{\lambda>0:(1.1)_{\lambda} \text { has a solution }\right\}>0 .
$$

It remains to show that for each $0<\lambda<\Lambda,(1.1)_{\lambda}$ has a solution $u$ with $I_{\lambda}(u)<0$. Let $0<\lambda<\Lambda$ and take $\bar{\lambda}$ such that $\lambda<\bar{\lambda}<\Lambda$ and (1.1) $\bar{\lambda}$ has a solution $\bar{u}$; this is clearly possible by the definition of $\Lambda$. One has, by the monotonicity of the family $f_{\lambda}$,

$$
-\Delta \bar{u}=f_{\bar{\lambda}}(x, \bar{u}) \geq f_{\lambda}(x, \bar{u}),
$$

which shows that $\bar{u}$ is an upper solution for $(1.1)_{\lambda}$. A previous argument involving the subdomain $\Omega_{1}$ from $\left(H_{\Omega_{1}}\right)$ shows that for $\varepsilon>0$ sufficiently small, $\varepsilon \varphi_{1}$ is a weak lower solution of $(1.1)_{\lambda}$ which satisfies $\varepsilon \varphi_{1} \leq \bar{u}$ in $\Omega$. Theorem 2.4 from [13] then yields the existence of a solution $u_{0} \in H_{0}^{1}(\Omega) \cap L^{\infty}(\Omega)$ of $(1.1)_{\lambda}$ which satisfies

$$
I_{\lambda}\left(u_{0}\right)=\min \left\{I_{\lambda}(u): u \in H_{0}^{1}(\Omega) \text { and } \varepsilon \varphi_{1} \leq u \leq \bar{u}\right\}
$$

Since by $\left(H_{\Omega_{1}}\right)$,

$$
I_{\lambda}\left(\varepsilon \varphi_{1}\right)=\frac{\varepsilon^{2}}{2} \int_{\Omega}\left|\nabla \varphi_{1}\right|^{2}-\int_{\Omega} F_{\lambda}\left(x, \varepsilon \varphi_{1}\right)<0
$$

for $\varepsilon$ sufficiently small (so that $\varepsilon \varphi_{1} \leq s_{1}$ ), one deduces $I_{\lambda}\left(u_{0}\right)<0$. This completes the proof of Theorem 2.1 
Proof of Theorem 2.2. We have to prove that for $\lambda$ sufficiently large, $(1.1)_{\lambda}$ has no solution. The subdomain $\tilde{\Omega}$ provided by $\left(H_{\tilde{\Omega}}\right)$ will be used here. Suppose that $(1.1)_{\lambda}$ admits a solution $u \in H_{0}^{1}(\Omega) \cap L^{\infty}(\Omega)$. Denoting by $\tilde{\varphi}$ the positive eigenfunction associated to the principal eigenvalue $\lambda_{1}(\tilde{m}, \tilde{\Omega})$ of $-\Delta$ on $H_{0}^{1}(\tilde{\Omega})$ for the weight $\tilde{m}$ and extending $\tilde{\varphi}$ by 0 on $\Omega \backslash \tilde{\Omega}$, one argues as in [9, p. 466] to get

$$
\int_{\Omega} \nabla u \nabla \tilde{\varphi}=\int_{\partial \tilde{\Omega}} u \frac{\partial \tilde{\varphi}}{\partial v}+\int_{\tilde{\Omega}} u(-\Delta \tilde{\varphi}) \leq \lambda_{1}(\tilde{m}, \tilde{\Omega}) \int_{\tilde{\Omega}} \tilde{m} u \tilde{\varphi} .
$$

On the other hand, by $\left(H_{\tilde{\Omega}}\right)$,

$$
\int_{\Omega} \nabla u \nabla \tilde{\varphi}=\int_{\Omega} f_{\lambda}(x, u) \tilde{\varphi} \geq h(\lambda) \int_{\tilde{\Omega}} \tilde{m} u \tilde{\varphi} .
$$

Since $\int_{\tilde{\Omega}} \tilde{m} u \tilde{\varphi}$ is $>0$, one deduces from 3.3 and 3.4 that $h(\lambda) \leq \lambda_{1}(\tilde{m}, \tilde{\Omega})$. The conclusion follows since $h(\lambda) \rightarrow \infty$ as $\lambda \rightarrow \infty$.

Proof of Theorem 2.3. We have to prove that $(1.1)_{\lambda}$ has at least one solution $u$ with $I_{\lambda}(u) \leq 0$ for $\lambda=\Lambda$. The continuity of $f_{\lambda}$ with respect to $\lambda$ as well as the fact that $(G)$ and $(A R)_{d}$ hold uniformly for $\lambda \in[r, R]$ will be used here. Let $\lambda_{k} \rightarrow \Lambda$ with $0<\lambda_{k}<\Lambda$ and $\lambda_{k}$ increasing, and let $u_{k}$ be a solution of $(1.1)_{\lambda_{k}}$ with $I\left(u_{k}\right)<0$.

We first show that the sequence $\left(u_{k}\right)$ remains bounded in $H_{0}^{1}(\Omega)$. Indeed, using $I_{\lambda_{k}}\left(u_{k}\right)<0$ and $(A R)_{d}$, one obtains

$$
\frac{\theta}{2}\left\|u_{k}\right\|^{2}-\int_{\Omega} u_{k} f_{\lambda_{k}}\left(x, u_{k}\right) \leq d \int_{\Omega} u_{k}^{\rho}+c_{1}
$$

for some constant $c_{1}$, where $\|v\|$ denotes $\left(\int_{\Omega}|\nabla v|^{2}\right)^{1 / 2}$. But $\int_{\Omega} u_{k} f_{\lambda_{k}}\left(x, u_{k}\right)=\left\|u_{k}\right\|^{2}$ by $(1.1)_{\lambda_{k}}$, and consequently

$$
\left(\frac{\theta}{2}-1\right)\left\|u_{k}\right\|^{2} \leq c_{2}\left\|u_{k}\right\|^{\rho}+c_{1}
$$

for some constant $c_{2}$. This implies the desired bound since $\theta>2$ and $\rho<2$.

Bootstrapping that bound using $(G)$, one sees in particular that for a subsequence, $u_{k} \rightarrow u$ in $H_{0}^{1}(\Omega) \cap C(\bar{\Omega})$. The bootstrapping here is the standard one when $\sigma<2^{*}-1$, and is based on [4] (see also [7]) when $\sigma=2^{*}-1$.

Clearly $u$ solves $-\Delta u=f_{\Lambda}(x, u)$ in $\Omega, u \geq 0$ in $\Omega$ and $u=0$ on $\partial \Omega$, and one has $I_{\Lambda}(u) \leq 0$. It remains to see that $u \not \equiv 0$. Assume by contradiction $u \equiv 0$. We will use $\left(H_{\Omega_{1}}\right.$ ) for $\lambda=\lambda_{1}$, the first element of the increasing sequence $\lambda_{k}$. Let as before $\Omega_{1}$ be the corresponding subdomain and $\varphi_{1}$ the positive eigenfunction associated to the principal eigenvalue $\lambda_{1}\left(\Omega_{1}\right)$ of $-\Delta$ on $H_{0}^{1}\left(\Omega_{1}\right)$. We have

$$
\int_{\Omega} \nabla u_{k} \nabla \varphi_{1}=\int_{\Omega_{1}} f_{\lambda_{k}}\left(x, u_{k}\right) \varphi_{1} \geq \int_{\Omega_{1}} f_{\lambda_{1}}\left(x, u_{k}\right) \varphi_{1} \geq \theta_{1} \int_{\Omega_{1}} u_{k} \varphi_{1}
$$


for $k$ sufficiently large (so that $0 \leq u_{k}(x) \leq s_{1}$ for $x \in \Omega_{1}$, which is possible since $u_{k} \rightarrow 0$ uniformly on $\bar{\Omega}$ ). On the other hand,

$$
\int_{\Omega_{1}} \nabla u_{k} \nabla \varphi_{1}=\int_{\partial \Omega_{1}} u_{k} \frac{\partial \varphi_{1}}{\partial v}+\int_{\Omega_{1}} u_{k}\left(-\Delta \varphi_{1}\right) \leq \lambda_{1}\left(\Omega_{1}\right) \int_{\Omega_{1}} u_{k} \varphi_{1},
$$

and a contradiction follows from (3.5), 3.6) since $\theta_{1}>\lambda_{1}\left(\Omega_{1}\right)$ and $\int_{\Omega_{1}} u_{k} \varphi_{1}>0$. This completes the proof of Theorem 2.3

Proof of Theorem 2.4. We have to prove the existence of a second solution of $(1.1)_{\lambda}$ for each $0<\lambda<\Lambda$. Fix such a $\lambda$. Proceeding exactly as at the end of the proof of Theorem 2.1 above, introducing $\bar{\lambda}, \bar{u}$ and considering the solution $u_{0}$ of $(1.1)_{\lambda}$ constructed there, we start by showing that

$$
\begin{gathered}
\underline{u}<u_{0}<\bar{u} \quad \text { in } \Omega, \\
\partial \underline{u} / \partial v>\partial u_{0} / \partial v>\partial \bar{u} / \partial v \quad \text { on } \partial \Omega,
\end{gathered}
$$

where $\underline{u}$ denotes $\varepsilon \varphi_{1}$, with $\varphi_{1}$ a positive principal eigenfunction of $-\Delta$ on $H_{0}^{1}\left(\Omega_{1}\right)$ (extended by 0 outside $\Omega_{1}$ ).

The inequalities of (3.7), (3.8) involving $\underline{u}$ and $u_{0}$ are obtained in the following way. Since $\underline{u}$ is the extension by 0 on $\Omega \backslash \Omega_{1}$ of a $C_{0}^{1}\left(\bar{\Omega}_{1}\right)$ function and since $u_{0}$ is a solution, these inequalities clearly hold on $\Omega \backslash \Omega_{1}$ and on $\partial \Omega \backslash \partial \Omega_{1}$ respectively. On the other hand $\underline{u} \not \equiv u_{0}$ in $\Omega_{1}$; moreover, using $\left(H_{0}\right)^{\prime}$, one gets for a suitable $B$,

$$
\begin{cases}-\Delta\left(u_{0}-\underline{u}\right) \geq f_{\lambda}\left(x, u_{0}\right)-f_{\lambda}(x, \underline{u}) \geq-B\left(u_{0}-\underline{u}\right) & \text { on } \Omega_{1}, \\ u_{0}-\underline{u} \geq 0 & \text { on } \Omega_{1} .\end{cases}
$$

Consequently, by the strong maximum principle, $u_{0}-\underline{u}>0$ in $\Omega_{1}$ and $\partial\left(u_{0}-\underline{u}\right) / \partial v<0$ on $\partial \Omega_{1}$. The proof of the inequalities in (3.7), 3.8) involving $u_{0}$ and $\bar{u}$ is simpler since both functions belong to $C_{0}^{1}(\bar{\Omega})$; the fact that $u_{0} \not \equiv \bar{u}$ in $\Omega$ here follows from $(M)$.

It follows from (3.7) and (3.8) that $\left\{u \in H_{0}^{1}(\Omega): \underline{u} \leq u \leq \bar{u}\right\}$ contains a $C_{0}^{1}(\bar{\Omega})$ neighborhood of $u_{0}$ and consequently, by $\left[3.1\right.$, $u_{0}$ is a local minimizer of $I_{\lambda}$ on $C_{0}^{1}(\bar{\Omega})$. Theorem 1 of [6] then shows that $u_{0}$ is also a local minimizer of $I_{\lambda}$ on $H_{0}^{1}(\Omega)$ (assumption $(G)$, with $\sigma \leq 2^{*}-1$, is used here).

The second solution will be constructed in the form $u_{0}+w$ where $u_{0}$ is the first solution above and $w$ satisfies

$$
\begin{cases}-\Delta w=g_{\lambda}(x, w) & \text { in } \Omega \\ w \neq \equiv 0 & \text { in } \Omega, \\ w=0 & \text { on } \partial \Omega\end{cases}
$$

where $g_{\lambda}(x, s):=f_{\lambda}\left(x, u_{0}(x)+s^{+}\right)-f_{\lambda}\left(x, u_{0}(x)\right)$. This is a device already considered in [1] for (1.2) with $a(x) \equiv b(x) \equiv 1$. Clearly any solution $w$ of 3.9 ) is $\geq 0$ (in fact, multiply by $-w^{-}$and conclude), and so, by the strong maximum principle and $\left(H_{0}\right)^{\prime}$, $w$ satisfies $w>0$ in $\Omega$ and $\partial w / \partial v<0$ on $\partial \Omega$. Consequently, $u_{0}+w$ will be a second 
solution of $(1.1)_{\lambda}$ which fulfills the requirements of Theorem 2.4 . Writing $G_{\lambda}(x, s):=$ $\int_{0}^{s} g_{\lambda}(x, t) d t$ and

$$
J_{\lambda}(w):=\frac{1}{2} \int_{\Omega}|\nabla w|^{2}-\int_{\Omega} G_{\lambda}(x, w),
$$

we are thus led to look for a nonzero critical point of $J_{\lambda}$ on $H_{0}^{1}(\Omega)$.

One easily verifies, using

$$
G_{\lambda}(x, s)=F_{\lambda}\left(x, u_{0}(x)+s^{+}\right)-F_{\lambda}\left(x, u_{0}(x)\right)-f_{\lambda}\left(x, u_{0}(x)\right) s^{+}
$$

and the fact that $u_{0}$ solves $(1.1)_{\lambda}$, that for $w \in H_{0}^{1}(\Omega)$,

$$
J_{\lambda}(w)=I_{\lambda}\left(u_{0}+w^{+}\right)-I_{\lambda}\left(u_{0}\right)+\frac{1}{2}\left\|w^{-}\right\|^{2} .
$$

It follows from (3.11) that 0 is a local minimizer of $J_{\lambda}$ on $H_{0}^{1}(\Omega)$, i.e., for some $r>0$,

$$
J_{\lambda}(0) \leq J_{\lambda}(w)
$$

for all $w \in B(0, r)$, the ball of center 0 and radius $r$ in $H_{0}^{1}(\Omega)$.

Assumption $(G)$ with $\sigma<2^{*}-1$ and $(A R)_{d}$ imply that $I_{\lambda}$ satisfies the (PS) condition on $H_{0}^{1}(\Omega)$, as shown in [9, p. 460]. On the other hand, one easily verifies that if $w_{k}$ is a (PS) sequence for $J_{\lambda}$ at level $c$, then $\left\|w_{k}^{-}\right\| \rightarrow 0$ and $u_{0}+w_{k}^{+}$is a (PS) sequence for $I_{\lambda}$ at level $c+I_{\lambda}\left(u_{0}\right)$. It follows that $J_{\lambda}$ satisfies the (PS) condition on $H_{0}^{1}(\Omega)$.

Now comes an alternative connected with (3.12). Either there exists $w \in B(0, r)$ with $w \neq 0$ and $J_{\lambda}(w)=0$, or the strict inequality holds in 3.12 for all $w \in B(0, r)$ with $w \neq 0$. In the first case this $w$ is a nonzero local minimizer for $J_{\lambda}$ and so a critical point of $J_{\lambda}$, and the proof is finished. In the second case, Theorem 5.10 from [8] applies to guarantee that for each $r>0$ sufficiently small,

$$
J_{\lambda}(0)<\inf \left\{J_{\lambda}(w): w \in H_{0}^{1}(\Omega) \text { and }\|w\|=r\right\}
$$

i.e. there is a "mountain range" around 0 . We aim at applying the mountain pass theorem. For that purpose we look for some $u_{2} \in H_{0}^{1}(\Omega)$ such that $J_{\lambda}\left(t u_{2}\right) \rightarrow-\infty$ as $t \rightarrow \infty$. Assumption $\left(H_{\Omega_{2}}\right)$ will be used here. In fact, as shown in [9. p. 462], $\left(H_{\Omega_{2}}\right)$ and $(A R)_{d}$ imply that for some $s_{3}$ and some $c>0$,

$$
F_{\lambda}(x, s) \geq c s^{\theta}
$$

for a.e. $x \in \Omega_{2}$ and all $s \geq s_{3}$, where $\theta>2$ comes from $(A R)_{d}$. This inequality clearly implies the same type of inequality for $G_{\lambda}$ :

$$
G_{\lambda}(x, s) \geq c^{\prime} s^{\theta}
$$

for some $s_{3}^{\prime}$ and $c^{\prime}>0$, and a.e. $x \in \Omega_{2}$ and all $s \geq s_{3}^{\prime}$. One then takes a smooth function $u_{2}$ with support in $\Omega_{2}$ and $u_{2} \geq 0, \not \equiv 0$. Calculating as in [9. p. 462], one finds that $J_{\lambda}\left(t u_{2}\right) \rightarrow-\infty$ as $t \rightarrow \infty$. The usual mountain pass theorem can thus be applied. This concludes the proof of Theorem 2.4 
Proof of Theorem 2.5. Fix $\lambda$ with $0<\lambda<\Lambda$. Proceeding exactly as at the beginning of the proof of Theorem 2.4, one has a first solution $u_{0}$ which is a local minimizer of $I_{\lambda}$ on $H_{0}^{1}(\Omega)$, and one is reduced to proving the existence of a solution $w$ of $(3.9)$, where $g_{\lambda}(x, s)$ now reads

$$
g_{\lambda}(x, s):=h_{\lambda}\left(x, u_{0}(x)+s^{+}\right)-h_{\lambda}\left(x, u_{0}(x)\right)+b(x)\left[\left(u_{0}(x)+s^{+}\right)^{p}-u_{0}(x)^{p}\right] .
$$

The associated functional $J_{\lambda}$ has again the form given in 3.10 , with now

$$
\begin{aligned}
G_{\lambda}(x, s):= & H_{\lambda}\left(x, u_{0}(x)+s^{+}\right)-H_{\lambda}\left(x, u_{0}(x)\right)-h_{\lambda}\left(x, u_{0}(x)\right) s^{+} \\
& +b(x)\left[\frac{\left(u_{0}(x)+s^{+}\right)^{p+1}-u_{0}(x)^{p+1}}{p+1}-u_{0}(x)^{p} s^{+}\right],
\end{aligned}
$$

where $H_{\lambda}(x, s):=\int_{0}^{1} h_{\lambda}(x, t) d t$. As before 0 is a local minimizer of $J_{\lambda}$ on $H_{0}^{1}(\Omega)$, and we are reduced to proving the existence of a nonzero critical point for $J_{\lambda}$.

Assume by contradiction that 0 is the only critical point of $J_{\lambda}$. Then, for some ball $B(0, r)$ in $H_{0}^{1}(\Omega)$,

$$
J_{\lambda}(0)<J_{\lambda}(w)
$$

for all $w \in B(0, r)$. The following lemma will be proved below.

Lemma 3.1. Assume 0 is the only critical point of $J_{\lambda}$. Then $J_{\lambda}$ satisfies the $(\mathrm{PS})_{c}$ condition for all levels $c$ with

$$
c<c_{0}:=S^{N / 2} /\left(N\|b\|_{\infty}^{(N-2) / 2}\right),
$$

where $S$ is the best Sobolev constant.

Using this lemma and Theorem 5.10 in [8] (which only requires the (PS) condition to hold at the level of the strict local minimum, here the level $J_{\lambda}(0)=0<c_{0}$ ), one deduces from 3.14 that 3.13 holds for all $r>0$ sufficiently small. We aim again at applying the mountain pass theorem. For this purpose we will show the existence of $u_{1} \in H_{0}^{1}(\Omega)$ such that $J_{\lambda}\left(u_{1}\right)<0$ and the infmax value of $J_{\lambda}$ over the family of all continuous paths from 0 to $u_{1}$ is $<c_{0}$. Once this is done, the usual mountain pass theorem yields the existence of a nonzero critical point for $J_{\lambda}$, a contradiction which will complete the proof of Theorem 2.5 .

To construct a $u_{1}$ as above, we consider as in [1] functions of the form $t \psi_{\mu}$ with $t>0$ and

$$
\psi_{\mu}(x):=d \zeta(x)\left(\frac{\mu}{\mu^{2}+\left|x-x_{0}\right|^{2}}\right)^{(N-2) / 2}
$$

where $\mu>0, x_{0}$ comes from assumption $(b), \zeta$ is a fixed smooth nonnegative function with $\zeta \equiv 1$ near $x_{0}$ and support in a small ball $B_{2}$ around $x_{0}$ (with $B_{2}$ chosen such that $\bar{B}_{2} \subset B_{1}$ and $b(x) \geq$ some $\varepsilon>0$ a.e. on $B_{2}$ ), and the normalizing constant $d>0$ is taken so that $\psi_{1}$ satisfies $-\Delta \psi_{1}=\psi_{1}^{(N+2) /(N-2)}$ near $x_{0}$. Since $h_{\lambda}$ satisfies $(G)$ with $\sigma<1$ (in fact $\sigma<p$ suffices in this part of the argument), one finds that for each $\mu>0, J_{\lambda}\left(t \psi_{\mu}\right) \rightarrow-\infty$ as $t \rightarrow \infty$, and consequently there exists $t=t_{\mu}>0$ such that $J_{\lambda}\left(t_{\mu} \psi_{\mu}\right)<0$. The following lemma implies that for $\mu$ sufficiently small, the infmax value of $J_{\lambda}$ over the family of all continuous paths from 0 to $u_{1}=t_{\mu} \psi_{\mu}$ is indeed $<c_{0}$. 
Lemma 3.2. One has

$$
\sup _{t>0} J_{\lambda}\left(t \psi_{\mu}\right)<c_{0}
$$

for $\mu>0$ sufficiently small.

The above two lemmas, to be proved below, complete the proof of Theorem 2.5 .

Proof of Lemma 3.1. Let $w_{n}$ be a (PS) $)_{c}$ sequence with $c<c_{0}$, i.e.

$$
\begin{gathered}
\frac{1}{2}\left\|w_{n}\right\|^{2}-\int_{\Omega} G_{\lambda}\left(x, w_{n}\right) \rightarrow c, \\
\int_{\Omega} \nabla w_{n} \cdot \nabla \varphi-\int_{\Omega} g_{\lambda}\left(x, w_{n}\right) \varphi \leq \varepsilon_{n}\|\varphi\|, \quad \forall \varphi \in H_{0}^{1}(\Omega),
\end{gathered}
$$

where $\varepsilon_{n} \rightarrow 0$. We first observe that $w_{n}$ remains bounded in $H_{0}^{1}(\Omega)$. This follows by multiplying 3.17) with $\varphi=u_{0}+w_{n}$ by $1 /(p+1)$ and subtracting from 3.16 ; the terms of power $p+1$ cancel and the remaining dominating term is $\left\|w_{n}\right\|^{2}$, which easily yields the boundedness of $w_{n}$. Note that the assumption that $h_{\lambda}$ satisfies $(G)$ with $\sigma<1$ is used in this argument. So, for a subsequence, $w_{n} \rightarrow w_{0}$ in $H_{0}^{1}(\Omega)$ and $w_{n} \rightarrow w_{0}$ in $L^{r}(\Omega)$ for any $r<2^{*}$. From (3.17) it follows that $w_{0}$ solves

$$
\begin{cases}-\Delta w=g_{\lambda}(x, w) & \text { in } \Omega \\ w=0 & \text { on } \partial \Omega\end{cases}
$$

and consequently, by the assumption of the lemma, $w_{0}=0$. We now go back to 3.17 with $\varphi=u_{0}+w_{n}$, multiply again by $1 /(p+1)$ and subtract from (3.16) to get

$$
\lim \left\|w_{n}\right\|^{2}=c N .
$$

There are two possibilities: either $c=0$ or $c \neq 0$. If $c=0$ then $w_{n}$ converges in $H_{0}^{1}(\Omega)$ by (3.18) and we are done. We will now see that $c \neq 0$ leads to a contradiction. For that purpose we deduce from 3.17 with $\varphi=w_{n}$ that

$$
\lim \left\|w_{n}\right\|^{2}=\lim \int_{\Omega} g_{\lambda}\left(x, w_{n}\right) w_{n}=\lim \int_{\Omega} b(x)\left(w_{n}^{+}\right)^{p+1} .
$$

By definition of $S$,

$$
\left\|w_{n}\right\|^{2} \geq S\left(\int_{\Omega}\left|w_{n}\right|^{2^{*}}\right)^{2 / 2^{*}} \geq \frac{S}{\|b\|_{\infty}^{2 / 2^{*}}}\left(\int_{\Omega} b(x)\left(w_{n}^{+}\right)^{2^{*}}\right)^{2 / 2^{*}},
$$

where the latter integral is $>0$ for $n$ sufficiently large (by 3.18, 3.19) and $c>0$ ). It follows from 3.18 -3.20 that

$$
c N \geq \frac{S}{\|b\|_{\infty}^{2 / 2^{*}}}(c N)^{2 / 2^{*}},
$$

i.e., $c \geq c_{0}$, as $c>0$. This contradicts 3.15 and completes the proof of Lemma 3.1 
Proof of Lemma 3.2 when $N \geq 4$. We start as in [1, p. 537] observing that for some positive constant $\alpha$,

$$
g_{\lambda}(x, s) \geq b(x)\left[\left(s^{+}\right)^{p}+\alpha\left(u_{0}(x)\right)^{p-1} s^{+}\right]
$$

a.e. on $B_{2}$. Note that the assumption that $h_{\lambda}$ is nondecreasing is used here; note also that $B_{2}$ was introduced just before the statement of Lemma 3.2 Consequently,

$$
J_{\lambda}\left(t \psi_{\mu}\right) \leq \frac{t^{2}}{2}\left\|\psi_{\mu}\right\|^{2}-\frac{t^{p+1}}{p+1} \int_{\Omega} b(x) \psi_{\mu}^{p+1}-\frac{t^{2}}{2} \alpha^{\prime}\left\|\psi_{\mu}\right\|_{2}^{2}
$$

for some other positive constant $\alpha^{\prime}$. Computing the maximum of the right-hand side for $t>0$ yields

$$
\sup _{t>0} J_{\lambda}\left(t \psi_{\mu}\right) \leq \frac{1}{N}\left[\left(\left\|\psi_{\mu}\right\|^{2}-\alpha^{\prime}\left\|\psi_{\mu}\right\|_{2}^{2}\right)^{+}\right]^{N / 2} /\left[\int_{\Omega} b(x) \psi_{\mu}^{2^{*}}\right]^{(N-2) / 2} .
$$

We will use the following estimates from [5] (see also [12, 14]) for $\mu \rightarrow 0$ :

$$
\begin{aligned}
&\left\|\psi_{\mu}\right\|^{2}=S^{N / 2}+O\left(\mu^{N-2}\right) \text { when } N \geq 3, \\
&\left\|\psi_{\mu}\right\|_{2^{*}}^{2^{*}}=S^{N / 2}+O\left(\mu^{N}\right) \text { when } N \geq 3, \\
&\left\|\psi_{\mu}\right\|_{2}^{2}= \begin{cases}k_{1} \mu^{2}+O\left(\mu^{N-2}\right) & \text { when } N \geq 5, \\
k_{2} \mu^{2}\left|\log \mu^{2}\right|+O\left(\mu^{2}\right) & \text { when } N=4,\end{cases}
\end{aligned}
$$

where $k_{1}, k_{2}$ are positive constants. To estimate the denominator in the right-hand side of 3.21, we call $b_{0}:=\|b\|_{\infty}$, introduce a ball $B_{\mu^{\delta}}=B\left(x_{0}, \mu^{\delta}\right)$ with $0<\delta<1$ to be determined later and write

$$
\int_{\Omega} b(x) \psi_{\mu}^{2^{*}}=\int_{B_{\mu^{\delta}}}\left(b(x)-b_{0}\right) \psi_{\mu}^{2^{*}}+\int_{\Omega \backslash B_{\mu^{\delta}}}\left(b(x)-b_{0}\right) \psi_{\mu}^{2^{*}}+b_{0}\left\|\psi_{\mu}\right\|_{2^{*}}^{2^{*}}
$$

Using assumption $(b)$ and 3.22 , one has

$$
\left|\int_{B_{\mu^{\delta}}}\left(b(x)-b_{0}\right) \psi_{\mu}^{2^{*}}\right| \leq \tilde{M} \mu^{\gamma \delta}\left[S^{N / 2}+O\left(\mu^{N}\right)\right]
$$

for some constant $\tilde{M}$. On the other hand, for some constant $C$,

$$
\left|\int_{\Omega \backslash B_{\mu^{\delta}}}\left(b(x)-b_{0}\right) \psi_{\mu}^{2^{*}}\right| \leq C \int_{\Omega \backslash B_{\mu^{\delta}}} \psi_{\mu}^{2^{*}}=O\left(\mu^{N(1-\delta)}\right),
$$

where the latter equality can be verified by using a Taylor expansion in

$$
\int_{\mu^{\delta}}^{\infty}\left[\mu /\left(\mu^{2}+r^{2}\right)^{N}\right] r^{N-1} d r
$$


Let us first consider the case $N \geq 5$. Using the above estimates in (3.21), one gets, for $\mu$ sufficiently small,

$$
\sup _{t>0} J_{\lambda}\left(t \psi_{\mu}\right) \leq \frac{S^{N / 2}}{N b_{0}^{(N-2) / 2}} \frac{\left[1-\alpha^{\prime \prime} \mu^{2}+O\left(\mu^{N-2}\right)\right]^{N / 2}}{\left[1+O\left(\mu^{\gamma \delta}\right)+O\left(\mu^{N(1-\delta)}\right)\right]^{(N-2) / 2}}
$$

with another positive constant $\alpha^{\prime \prime}$. Since $\gamma>2^{*}$, one can find $\delta$ such that $\gamma \delta>2$ and $N(1-\delta)>2$. It follows that the quotient $[\cdots]^{N / 2} /[\cdots]^{(N-2) / 2}$ in 3.23 is $<1$ for $\mu$ sufficiently small. This proves the lemma when $N \geq 5$.

When $N=4$ the bracket $[\cdots]^{N / 2}$ in 3.23 now reads

$$
\left[1-\alpha^{\prime \prime} \mu^{2}\left|\log \mu^{2}\right|+O\left(\mu^{2}\right)\right]^{N / 2},
$$

and the same argument as above, using $\gamma \geq 2^{*}$, yields the conclusion.

Proof of Lemma 3.2 when $N=3$. We again start as in [1 p. 537] to reach here

$$
J_{\lambda}\left(t \psi_{\mu}\right) \leq \frac{t^{2}}{2}\left\|\psi_{\mu}\right\|^{2}-\frac{t^{6}}{6} \int_{\Omega} b(x) \psi_{\mu}^{6}-\frac{t^{5}}{5} \alpha\left\|\psi_{\mu}\right\|_{5}^{5}
$$

for some positive constant $\alpha$. The maximum of the right-hand side for $t>0$ is achieved for $t_{0}=t_{0}(\mu)$ satisfying

$$
\left\|\psi_{\mu}\right\|^{2}=\left(\int_{\Omega} b(x) \psi_{\mu}^{6}\right) t_{0}^{4}+\alpha\left\|\psi_{\mu}\right\|_{5}^{5} t_{0}^{3} .
$$

In addition to (3.22) we will use

$$
\left\|\psi_{\mu}\right\|_{5}^{5}=k \mu^{1 / 2}+O\left(\mu^{5 / 2}\right)
$$

with $k$ a positive constant (cf. [1]). We will also use

$$
\int_{\Omega} b(x) \psi_{\mu}^{6}=b_{0} S^{3 / 2}+O\left(\mu^{\gamma \delta}\right)+O\left(\mu^{3(1-\delta)}\right),
$$

which is obtained as in the proof for $N \geq 4$.

Using 3.26, 3.27) together with 3.22, one deduces from 3.25 that

$$
t_{0}(\mu)=\frac{1}{b_{0}^{1 / 4}}-\frac{k}{4 b_{0} S^{3 / 2}} \mu^{1 / 2}+o\left(\mu^{1 / 2}\right)
$$

provided $\delta$ is chosen so that $\gamma \delta>1 / 2$ and $3(1-\delta)>1 / 2$, which is possible since $\gamma>3 / 5$. It then follows from 3.24 that

$$
\sup _{t>0} J_{\lambda}\left(t \psi_{\mu}\right) \leq \frac{S^{3 / 2}}{3 b_{0}^{1 / 2}}-\frac{k}{5 b_{0}^{5 / 4}} \mu^{1 / 2}+o\left(\mu^{1 / 2}\right)<\frac{S^{3 / 2}}{3 b_{0}^{1 / 2}}
$$

for $\mu$ sufficiently small. This is the conclusion of Lemma 3.2 when $N=3$. 
Proof of Theorem 2.6. The only difference with the proof of Theorem 2.5 occurs at the beginning of the proof of Lemma 3.1, at the point where one shows that any (PS) $)_{c}$ sequence is bounded.

The argument to prove that any sequence $w_{n}$ satisfying 3.16 and 3.17 is bounded here goes as follows. First observe that in our situation, $H_{\lambda}(x, s) \geq 0$ and so $\theta$ in the condition $(A R)_{d}$ for $h_{\lambda}$ can always be chosen such that $2<\theta<p+1$. We will estimate

$$
\Phi\left(w_{n}\right):=J_{\lambda}\left(w_{n}\right)-\frac{1}{\theta} J_{\lambda}^{\prime}\left(w_{n}\right)\left(u_{0}+w_{n}\right)
$$

By (3.16) and (3.17), one has, for some constant $C$,

$$
\Phi\left(w_{n}\right) \leq C+\frac{\varepsilon_{n}}{\theta}\left\|u_{0}+w_{n}\right\|
$$

On the other hand, expanding $\Phi\left(w_{n}\right)$, one obtains

$$
\begin{aligned}
\Phi\left(w_{n}\right)= & \left(\frac{1}{2}-\frac{1}{\theta}\right)\left\|w_{n}\right\|^{2}-\int_{\Omega}\left[H_{\lambda}\left(x, u_{0}+w_{n}^{+}\right)-\frac{1}{\theta} h_{\lambda}\left(x, u_{0}+w_{n}^{+}\right)\left(u_{0}+w_{n}^{+}\right)\right] \\
& -\left(\frac{1}{p+1}-\frac{1}{\theta}\right) \int_{\Omega} b(x)\left(u_{0}+w_{n}^{+}\right)^{p+1}+A_{n}
\end{aligned}
$$

where $A_{n}$ is a first order term, i.e. satisfies $\left\|A_{n}\right\| \leq c_{1}+c_{2}\left\|w_{n}\right\|$ for some constants $c_{1}, c_{2}$. Combining (3.28) and 3.29) gives

$$
\begin{aligned}
\left(\frac{1}{2}-\frac{1}{\theta}\right)\left\|w_{n}\right\|^{2}= & \int_{\Omega}\left[H_{\lambda}\left(x, u_{0}+w_{n}^{+}\right)-\frac{1}{\theta} h_{\lambda}\left(x, u_{0}+w_{n}^{+}\right)\left(u_{0}+w_{n}^{+}\right)\right] \\
& +\left(\frac{1}{p+1}-\frac{1}{\theta}\right) \int_{\Omega} b(x)\left(u_{0}+w_{n}^{+}\right)^{p+1}+A_{n}^{\prime},
\end{aligned}
$$

for another first order term $A_{n}^{\prime}$. Using $(A R)_{d}, 2<\theta<p+1$ and $b(x) \geq 0$, one easily concludes that $w_{n}$ remains bounded. The proof of Theorem 2.6 is thus complete.

\section{Applications}

In this section we will see how the previous theorems apply to problems 1.2 and 1.3 . We start with 1.2, where $I_{\lambda}(u)$ now reads

$$
I_{\lambda}(u)=\frac{1}{2} \int_{\Omega}|\nabla u|^{2}-\frac{\lambda}{q+1} \int_{\Omega} a(x)\left(u^{+}\right)^{q+1}-\frac{1}{p+1} \int_{\Omega} b(x)\left(u^{+}\right)^{p+1} .
$$

Theorem 4.1. Let $0 \leq q<1<p$ and assume that $a, b \in L^{\infty}(\Omega)$ with

(i) $a(x) \geq 0$ a.e. $x$ in $\Omega$,

(ii) $a(x) \geq \varepsilon_{1}>0$ a.e. on some ball $B_{1}$.

Then there exists $0<\Lambda \leq \infty$ such that problem 1.2] has at least one solution $u$ (with $\left.I_{\lambda}(u)<0\right)$ for $0<\lambda<\Lambda$ and no solution for $\lambda>\Lambda$. If in addition 
(iii) $b(x) \geq 0$ a.e. on some ball $B_{2}$, with $a(x) b(x) \not \equiv 0$ on $B_{2}$,

then $\Lambda<\infty$. Moreover, if in addition $p \leq 2^{*}-1$, then problem (1.2) has at least one solution $u$ (with $\left.I_{\lambda}(u) \leq 0\right)$ for $\lambda=\Lambda$.

Note that $\Lambda$ can be $\infty$ in the first part of Theorem 4.1. This happens for instance if $b(x) \equiv-1$ (cf. the observation following Theorem 2.1).

Proof of Theorem 4.1. It suffices to verify the hypotheses of Theorems 2.1, 2.2 and 2.3 $(H)$ and $\left(H_{0}\right)$ are obvious, by (i). In $\left(H_{e}\right)$ one takes $g(s)=\lambda\|a\|_{\infty} s^{q}+\|b\|_{\infty} s^{p}$ with $\lambda$ sufficiently small. $\left(H_{\Omega_{1}}\right)$ follows from (ii). At this stage Theorem 2.1 yields the first part of Theorem 4.1 . On the other hand, $\left(H_{\tilde{\Omega}}\right)$ follows from (iii) by applying Lemma 3.6 from [9]. Theorem 2.2 thus yields the second part of Theorem 4.1 Finally, $(G)$ is obvious when $p \leq 2^{*}-1$, and $(A R)_{d}$ follows as in [9. p. 457] by taking $\theta=p+1, \rho=q+1$, $d=R(\theta /(p+1)-1)\|a\|_{\infty}$ and $s_{0}=0$. (Recall that $\lambda \in[r, R]$ in $(A R)_{d}$.) The last part of Theorem 4.1 thus follows from Theorem 2.3

Theorem 4.2. Let $0 \leq q<1<p$ and assume that $a, b \in L^{\infty}(\Omega)$ with (i) and (ii) above. Assume in addition either $p<2^{*}-1$ and

(iv) $b(x) \geq \varepsilon_{2}>0$ a.e. on some ball $B_{2}$,

or $p=2^{*}-1$ and condition (b) of Theorem 2.5 for $b(x)$. Then problem (1.2) has at least two solutions $u, v$ for $0<\lambda<\Lambda$, with $u<v$ in $\Omega$, $\partial u / \partial v>\partial v / \partial v$ on $\partial \Omega$ and $I_{\lambda}(u)<0$.

Note that $(b)$ is a stronger condition than (iv). Note also that $b(x)$ above is allowed to change sign in $\Omega$.

Proof of Theorem 4.2. It suffices to verify the hypotheses of Theorems 2.4 and 2.5 . As observed in the proof of Theorem 4.1, the hypotheses of Theorem 2.1 follow from (i) and (ii), and $(A R)_{d}$ can be verified as in [9, p. 457]. Moreover, $\left(H_{0}^{\prime}\right)$ and $(M)$ are obvious. Theorem 2.4 thus applies when $p<2^{*}-1$. In the critical case $p=2^{*}-1$, Theorem 2.5 clearly applies.

We now turn to problem 1.3$)$. The functional $I_{\lambda}(u)$ here reads

$$
I_{\lambda}(u)=\frac{1}{2} \int_{\Omega}|\nabla u|^{2}-\frac{\lambda}{p+1} \int_{\Omega} c(x)\left(u^{+}+1\right)^{p+1} .
$$

Theorem 4.3. Let $p>1$ and assume that $c \in L^{\infty}(\Omega)$ with

$$
c(x) \geq 0 \quad \text { a.e. in } \Omega \quad \text { and } \quad c(x) \geq \varepsilon>0 \quad \text { a.e. on some ball } B .
$$

Then there exists $0<\Lambda<\infty$ such that problem $(1.3)$ has at least one solution $u$ (with $\left.I_{\lambda}(u)<0\right)$ for $0<\lambda<\Lambda$ and no solution for $\lambda>\Lambda$. Moreover, if $p \leq 2^{*}-1$, then problem $(1.3)$ has at least one solution $u$ (with $\left.I_{\lambda}(u) \leq 0\right)$ for $\lambda=\Lambda$. 
Proof. Theorems 2.1 2.3 easily apply to yield the desired conclusions. In the verification of $\left(H_{e}\right)$ one can take $g(s)=\lambda\|c\|_{\infty}(s+1)^{p}$. In the verification of $(A R)_{d}$ one has

$$
\theta F_{\lambda}(x, s)-s f_{\lambda}(x, s) \leq \lambda c(x)(s+1)^{p}\left[\left(\frac{\theta}{p+1}-1\right)(s+1)+1\right]
$$

and so, if we choose $\theta$ with $2<\theta<p+1$, the right-hand side of 4.2 is $\leq 0$ for $s$ sufficiently large, which yields $(A R)_{d}$ with $d=0$.

Theorem 4.4. Let $p>1$ and assume that $a \in L^{\infty}(\Omega)$ with (4.1). Assume in addition either $p<2^{*}-1$, or $p=2^{*}-1$ and condition (b) of Theorem 2.5 holds for $a(x)$. Then problem (1.3) has at least two solutions $u, v$ for $0<\lambda<\Lambda$, with $u<v$ in $\Omega$, $\partial u / \partial v>\partial v / \partial v$ on $\partial \Omega$ and $I_{\lambda}(u)<0$.

Proof. The subcritical case $p<2^{*}-1$ follows immediately from Theorem 2.4. The critical case $p=2^{*}-1$ requires more care because the right-hand side of $(1.3)$ is not written in the form 2.1. . However, $u$ solves 1.3 for $\lambda$ if and only if $v=\lambda^{\Gamma /}(p-1) u$ solves

$$
\begin{cases}-\Delta v=c(x)(v+\mu)^{p} & \text { in } \Omega, \\ v>0 & \text { in } \Omega, \\ v=0 & \text { on } \partial \Omega,\end{cases}
$$

for $\mu=\lambda^{1 /(p-1)}$. It follows in particular that 4.3 has at least one solution for $\mu<$ $\Lambda^{1 /(p-1)}$ and no solution for $\mu>\Lambda^{1 /(p-1)}$. We aim at applying Theorem 2.6 to 4.3 . For this purpose, we write

$$
c(x)(s+\mu)^{p}=h_{\mu}(x, s)+c(x) s^{p},
$$

where $h_{\mu}(x, s)=c(x)\left[(s+\mu)^{p}-s^{p}\right]$. A simple application of the mean value theorem shows that $h_{\mu}(x, s)$ satisfies $(G)$ with $\sigma=p-1$, and a calculation similar to 4.2 . shows that it satisfies $(A R)_{d}$ with $d=0$. The other hypotheses of Theorem 2.6 are easily verified, in the same way as they were verified earlier for $(1.3)$. It follows that (4.3) admits a second solution for $\mu<\Lambda^{1 /(p-1)}$, with negative energy. Finally, one observes that the energy of the corresponding solution of $(1.3)$ is also negative.

\section{References}

[1] Ambrosetti, A., Brezis, H., Cerami, G.: Combined effects of concave and convex nonlinearities in some elliptic problems. J. Funct. Anal. 122, 519-543 (1994) Zbl 0805.35028 MR 1276168

[2] Ambrosetti, A., Rabinowitz, P.: Dual variational methods in critical point theory and applications. J. Funct. Anal. 14, 349-381 (1973) Zbl 0273.49063 MR 0370183

[3] Bandle, C.: Existence theorems, qualitative results and a priori bounds for a class of nonlinear Dirichlet problems. Arch. Rat. Mech. Anal. 58, 219-238 (1975) Zbl 0335.35046 MR 0454336

[4] Brezis, H., Kato, T.: Remarks on the Schrödinger operator with singular complex potentials. J. Math. Pures Appl. 58, 137-151 (1979) Zbl 0408.35025 MR 0539217 
[5] Brezis, H., Nirenberg, L.: Positive solutions of nonlinear elliptic equations involving critical Sobolev exponents. Comm. Pure Appl. Math. 36, 437-477 (1983) Zbl 0541.35029 MR 0709644

[6] Brezis, H., Nirenberg, L.: $H^{1}$ versus $C^{1}$ local minimizers. C. R. Acad. Sci. Paris 317, 465-472 (1993) Zbl 0803.35029 MR 1239032

[7] de Figueiredo, D.: On the superlinear Ambrosetti-Prodi problem. Nonlinear Anal. 8, 655-665 (1984) Zbl 0554.35045 MR 0746723

[8] de Figueiredo, D.: Lectures on the Ekeland Variational Principle with Applications and Detours. Tata Inst. Fund. Res. Lectures Math. Phys. 81, Springer (1989) Zbl 0688.49011 MR 1019559

[9] de Figueiredo, D., Gossez, J.-P., Ubilla, P.: Local superlinearity and sublinearity for indefinite semilinear elliptic problems. J. Funct. Anal. 199, 452-467 (2003) Zbl 1034.35042 MR 1971261

[10] de Figueiredo, D., Lions, P. L.: On pairs of positive solutions for a class of semilinear elliptic problems. Indiana Univ. Math. J. 34, 591-606 (1985) Zbl 0587.35033 MR 0794577

[11] Gazzola, F., Malchiodi, A.: Some remarks on the equation $-\Delta u=\lambda(1+u)^{p}$ for varying $\lambda, p$ and varying domains. Comm. Partial Differential Equations 27, 809-845 (2002) Zbl 1010.35042 MR 1900564

[12] Ghoussoub, N.: Duality and Perturbation Methods in Critical Point Theory. Cambridge Tracts in Math. 107, Cambridge Univ. Press (1993) Zbl 0790.58002 MR 1251958

[13] Struwe, M.: Variational Methods. Springer (1990) Zbl 0746.49010 MR 1078018

[14] Willem, M.: Minimax Theorems. Progr. Nonlinear Differential Equations Appl. 24, Birkhäuser, Boston (1996) Zbl 0856.49001 MR 1400007 